\title{
Full Mouth Rehabilitation of a Patient with Severely Worn Dentition - A Systematic Clinical Approach
}

\author{
Dr. Rajul Vivek ${ }^{1}$, Dr. T.P. Chaturvedi ${ }^{2}$, Dr. Atul Bhatnagar ${ }^{3}$ Dr. Romesh Soni \\ ${ }^{4}$, Dr. Harakh Chand Baranwal ${ }^{5}$ \\ ${ }^{I}$ (Ex. Service Senior Resident, Department of Prosthodontics, Faculty of Dental Sciences, IMS, Banaras Hindu \\ University) \\ ${ }_{2}^{2}$ (Professor \& Dean, Department of Orthodontics, Faculty of Dental Sciences, IMS, Banaras Hindu \\ University) \\ ${ }^{3}$ (Associate Professor, Department of Prosthodontics, Faculty of Dental Sciences, IMS, Banaras Hindu \\ University) \\ ${ }^{4}$ (Assistant Professor, Department of Prosthodontics, Faculty of Dental Sciences, IMS, Banaras Hindu \\ University) \\ ${ }^{5}$ (Associate Professor, Department of Conservative Dentistry \& Endodontics, Faculty of Dental Sciences, IMS, \\ Banaras Hindu University)
}

\section{Introduction}

Full mouth rehabilitation cases are one of the most difficult cases to manage in dental practice. This is because such cases involve not only replacement of the lost tooth structure but also restoring the lost vertical dimensions. ${ }^{1}$ Full mouth reconstruction is basically a set of procedures that are aimed at correcting an improper bite position as well as restoring chipped or worn out teeth. Full mouth rehabilitation is done where severe tooth wearing present.

Severe tooth wear is a potential threat for dentition and masticatory function. Many factors may combine to produce the worn dentition, and the etiology often remains unidentified.$^{2}$ The gradual wear of the occlusal surfaces of teeth is a normal process during the lifetime of a patient. However, excessive occlusal wear can result in pulpal pathology, occlusal disharmony, impaired function, and esthetic disfigurement. ${ }^{3}$ Tooth wear can be classified as attrition, abrasion, and erosion depending on its cause. A differential diagnosis is not always possible because, in many situations, there exists a combination of these processes. ${ }^{4}$ Therefore, it is important to identify the factors that contribute to excessive wear and to evaluate alteration of the VDO caused by the worn dentition. ${ }^{5}$

Full mouth rehabilitation is a challenging treatment modality that enhances the appearance of the patient and corrects imperfections in the occlusion. ${ }^{6}$ The complexity in treating full mouth rehabilitation cases is not only because of its long treatment time but also at times the lack of clarity in the treatment objective. A case has to be treated not only by correcting worn out, broken or discolored teeth but also requires treating the oral cavity holistically. Every patient with extreme tooth wear has unique treatment needs. ${ }^{7}$ This article reports a sequence of Full mouth rehabilitation in a patient with completely worn dentition.

\section{Case Report}

A 52 year woman was referred for the treatment of her severely worn dentition. Her chief complaint was that she could not eat anything because her teeth were worn too much. On detailed history she told teeth are gradually becoming small over the years, generalized sensitivity, pain in lower front teeth for last one month.

Extraoral examination revealed no facial asymmetry or muscle tenderness. The mandibular movements were normal. Intraoral examination revealed overclosure, generalized severely worn dentition (Figure.1). The patient's facial appearance showed signs of collapsed VDO. The preoperative interocclusal rest space (IRS) was $5.8 \mathrm{~mm}$. Periodontal and soft-tissue examination showed pocket depth of $<2 \mathrm{~mm}$ and no mobility of remaining teeth(Figure.2,3). Radiographic evaluation showed excellent bone support for all teeth (Figure4). The patient was explained about the treatment plan. The aim of the treatment was to improve esthetics and restore occlusion so as to achieve optimum oral health.

Two sets of irreversible hydrocolloid impressions were made in stock trays (Figure.5a,5b). The patients existing Vertical Dimension of occlusion (VDO) and Physiologic rest position (PRP) were recorded. Diagnostic casts were mounted on a Hanau semi-adjustable articulator using a face-bow transfer and a centric relation record was made at the existing VDO. A diagnostic wax up was done at the estimated restored VDO (Figure.6). The patient's interocclusal rest space (IRS) was determined by taking the difference between the patient's existing PRP and the VDO. The diagnostic wax up of teeth was done with an estimated increased in VDO of 
$3 \mathrm{~mm}$ to achieve a mutually protected occlusion (Figure.7). Permissive occlusal splint was constructed to allow the patient to adapt to the new vertical dimension.

The patient was then referred to endodontic department for root canal therapy with $\# 17, \# 16, \# 15, \# 14$, $\# 21, \# 22, \# 23, \# 31, \# 32, \# 33$, and \#44. Tooth preparations were done (Figure.8) and provisional restorations were cemented with zinc oxide luting cement. For three months, interim restorations were adjusted, and used as a guide for the definitive oral rehabilitation. During this period, the patient's condition and functions, such as muscle tenderness, discomfort of TMJ, mastication, range of the mandibular movements, swallowing, and speech, were evaluated. Improvement in mastication, speech, and facial esthetics confirmed the patient's tolerance to the new mandibular position with the restored VDO. The final prosthesis was constructed after the provisional has been used for 3 months(Figure.9) .After insertion of interim prosthesis, the patient reported a slight difference in her pronunciation of the ch, jh, $\mathrm{s}^{\mathrm{ee}}$ sound; however, this problem resolved itself after 15 days. The patient reported with no muscular or temporomandibular joint discomfort. Follow-up treatments were done to evaluate the patient's comfort, arch form and potential VDO problems. The PRP was accommodated, and the patient presented with a $4 \mathrm{~mm}$ IRS within a few months. Impression for the mandibular and maxillary final prosthesis was made using polyvinyl silicones with a double mix double impression technique. Cast of the prepared teeth were mounted on the Hanau articulator and a centric relation record was made at the predetermined restored VDO. Finally the restoration were polished and cemented with glass ionomer cement. Oral hygiene instruction and regular check-up were administered.

\section{Discussion}

In 1984, Turner classified the treatment of a severely worn dentition by the amount of the loss of VDO and available space to restore. His classification and conventional treatment, which includes raising VDO with multiple crown-lengthening procedures, have been widely used up to present. However, the etiology of tooth wear is multifactorial, and clinical controlled trials of restorative and prosthodontic approaches are limited in quantity and quality ${ }^{8}$

In the treatment of severely worn teeth, equal-intensity centric occlusal contacts on all teeth should be accomplished. An anterior guidance should also be established in harmony with normal functional jaw movements and all posterior teeth discluded immediately during any eccentric jaw movement.

Full mouth rehabilitation involves the procedures necessary to produce a healthy, esthetic, well functioning masticatory system. Treating the full mouth rehabilitation patients involves not only then esthetic coverage restorations but also the meticulous planning of occlusion .

The provisional restorations play a critical role in the successful treatment of the full mouth rehabilitation patient. Good quality provisional restorations are essential to achieve predictability with comprehensive cases involving severe parafunctional habits. The provisional restorations should be esthetic and also fulfill the functions so that the effect can be followed in the temporary before making the final restoration.

In previous literature, the wearing time of provisional crown are various. The trial period of intensive fixed provisional prostheses is $2-6$ months. ${ }^{3,10,11,12,13}$ In this case; the patient was carefully monitored for 3 month to evaluate the adaptation of the provisional restoration.$^{14}$

The trial period is relatively same for this case as in the other case report, but discomfort, wear, and muscle fatigue were not observed during that period. The increase of VDO was determined

not by standardized esthetic golden proportion of anterior teeth but by patient's physiologic factor like interocclusal rest space and speech. If the increase of VDO was decided arbitrarily without close evaluation, multiple complications would happen and longer treatment period might be needed. Depending on the patient's situation and adaptation ability, the interim period can be modified, and the careful evaluation and monitoring may shorten the overall treatment duration.

\section{Conclusion}

Severe tooth wear is frequently multifactorial and variable. Restoration of worn dentition is a challenge to a prosthodontist. A combination of "mechanical, biological, esthetic factor is mandatory, for Full mouth rehabilitation Successful management is a subject of interest in dentistry. A critical aspect is to determine the occlusal vertical dimension (OVD) and a systematic approach that can lead to a predictable and favorable treatment prognosis. Management of patients with worn dentition is complex and difficult. Accurate clinical and radiographic examinations, a diagnostic wax-up, and determining OVD are crucial.

\section{References}

[1]. Gopal Y, Mallabadi R et al, Full Mouth Rehabilitation Journal of Clinical and Diagnostic Research 2007 June,1(3): 143-146

[2]. A. J. R. Crothers, "Tooth wear and facial morphology," Journal of Dentistry, vol. 20, no. 6, pp. 333-341, 1992.

[3]. Turner KA, Missirlian DM. Restoration of the extremely worn dentition. J Prosthet Dent 1984; 52:467-74.

[4]. Smith BG. Toothwear: aetiology and diagnosis. Dent Update 1989; 16:204-12. 
[5]. Prasad S, Kuracina J, Monaco EA Jr. Altering occlusal vertical dimension provisionally with base metal onlays: a clinical report. J Prosthet Dent 2008; 100:338-42.

[6]. Dawson PE. Functional occlusion from TMJ to smile design. Mosby St. Louis, Elsevier. 2007:18-26, 27-32, 75-83, 429-52.

[7]. Binkley TK, Binkley CJ. A practical approach to full mouth rehabilitation. J Prosthet Dent 1987; 57(3): 261-6.

[8]. Turner KA, Missirlian DM. Restoration of the extremely worn dentition. J Prosthet Dent 1984; 52:467-74.

[9]. Rivera-Moreles WC, Mohl ND. Restoration of vertical dimension of occlusion in the severely worn dentition. Dent Clin North Am 1992;36(3):651-64

[10]. Jahangiri L, Jang S. Onlay partial denture technique for assessment of adequate occlusal vertical dimension: a clinical report. J Prosthet Dent 2002; 87:1-4.

[11]. Hemmings KW, Darbar UR, Vaughan S. Tooth wear treated with direct composite restorations at an increased vertical dimension: results at 30 months. J Prosthet Dent 2000; 83:287-93.

[12]. Sato S, Hotta TH, Pedrazzi V. Removable occlusal overlay splint in the management of tooth wear: a clinical report. J Prosthet Dent 2000; 83:392-5.

[13]. Brown KE. Reconstruction considerations for severe dental attrition. J Prosthet Dent 1980;44:384-8

[14]. Yunus N, Abdullah H, Hanapiah F. The use of implants in the occlusal rehabilitation of a partially edentulous patient: a clinical report. J Prosthet Dent 2001; 85:540-3.

\section{Legends-}

Figure.1Oral examination- A Frontal view

Figure.2Maxillary occlusal view

Figure.3Mandibular occlusal view

Figure.4 Panoramic radiograph

Figure.5 (a) Maxillary Diagnostic impression

Figure.5 (b) Mandibular Diagnostic impression

Figure.6 Diagnostic wax-up.

Figure.7Group Function occlusion

Figure. 8 Tooth Preparation

Figure.9Definitive prosthesis

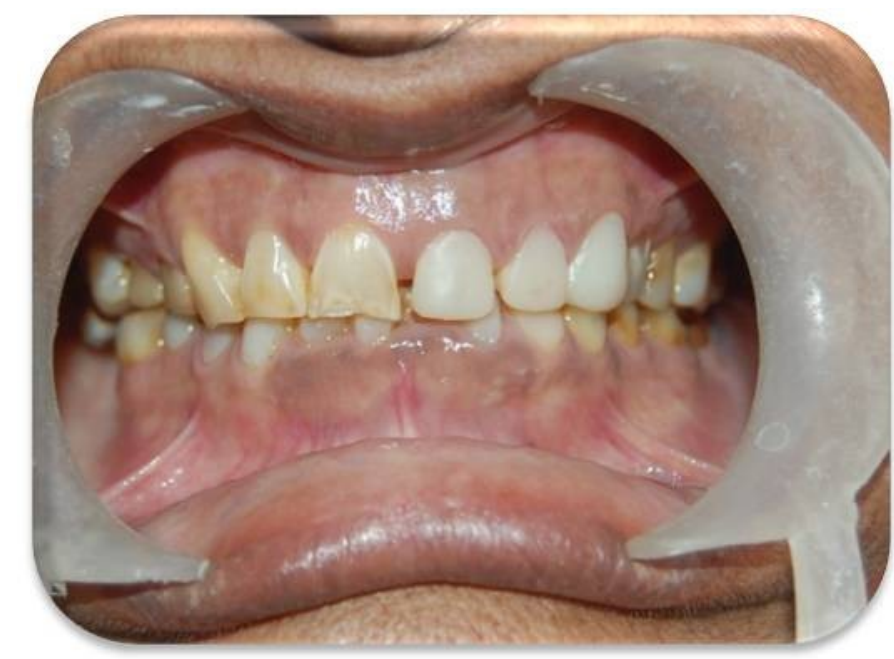

Figure.1Oral examination- A Frontal view

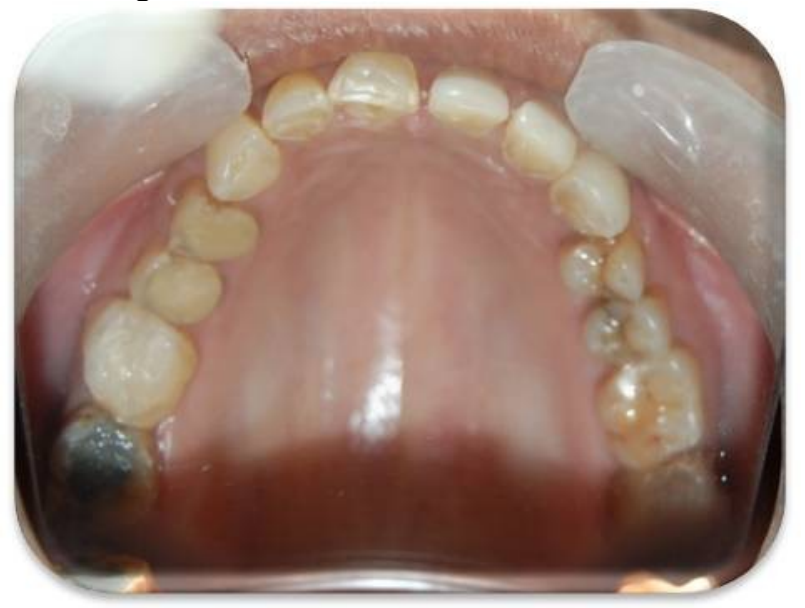

Figure.2Maxillary occlusal view 


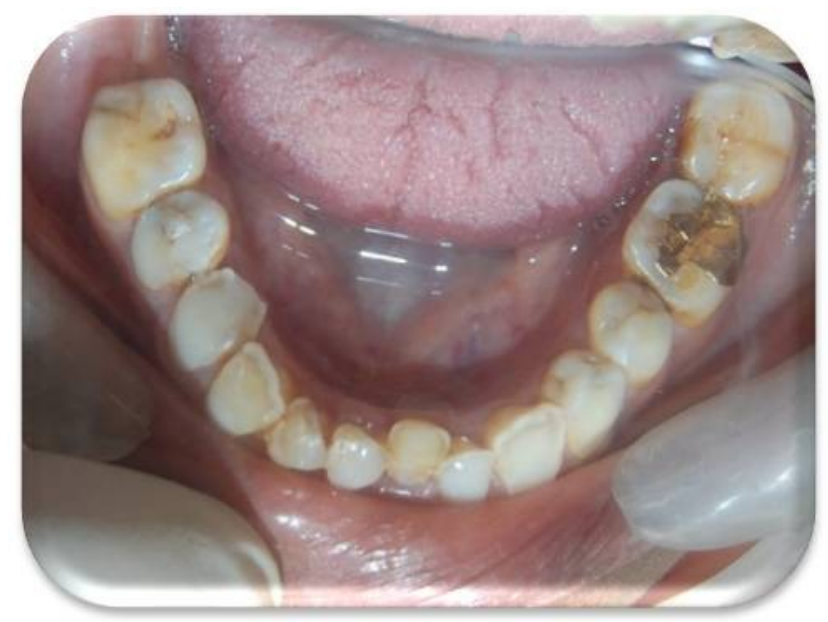

Figure.3Mandibular occlusal view

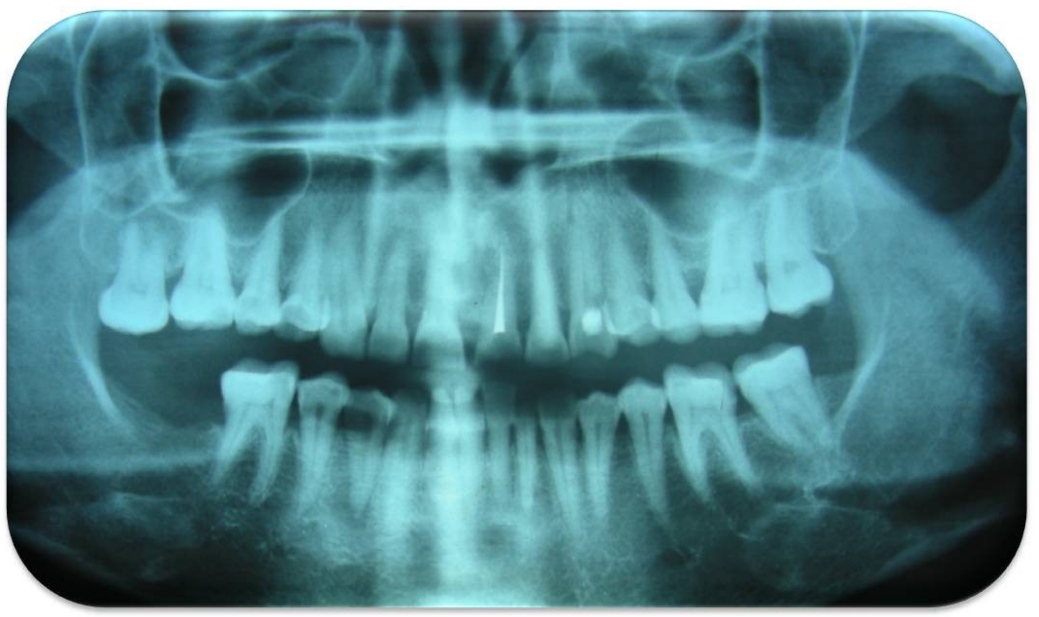

Figure.4 Panoramic radiograph

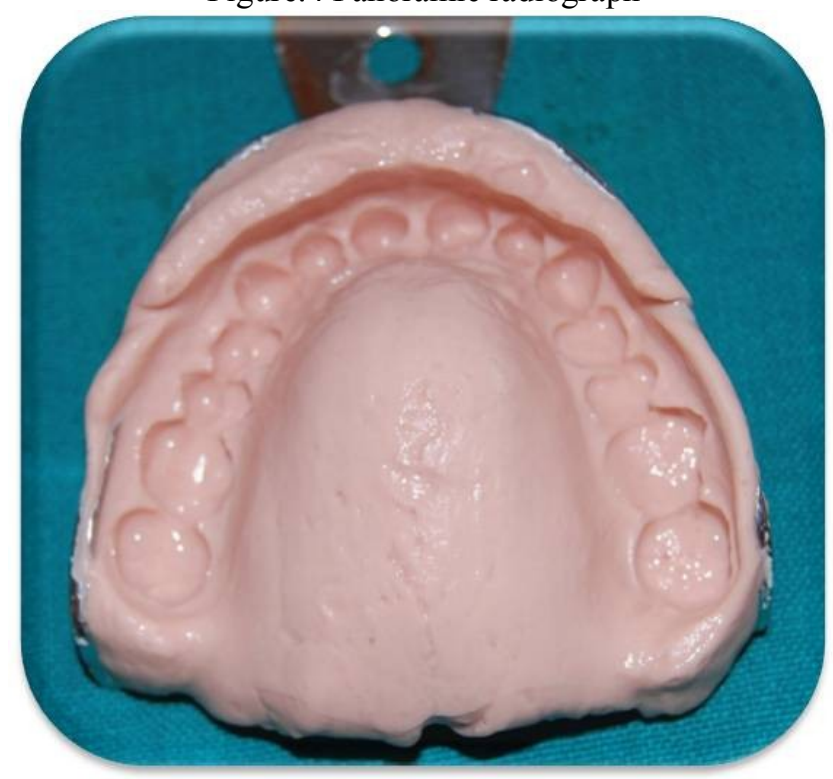

Figure.5 (a) Maxillary Diagnostic impression 


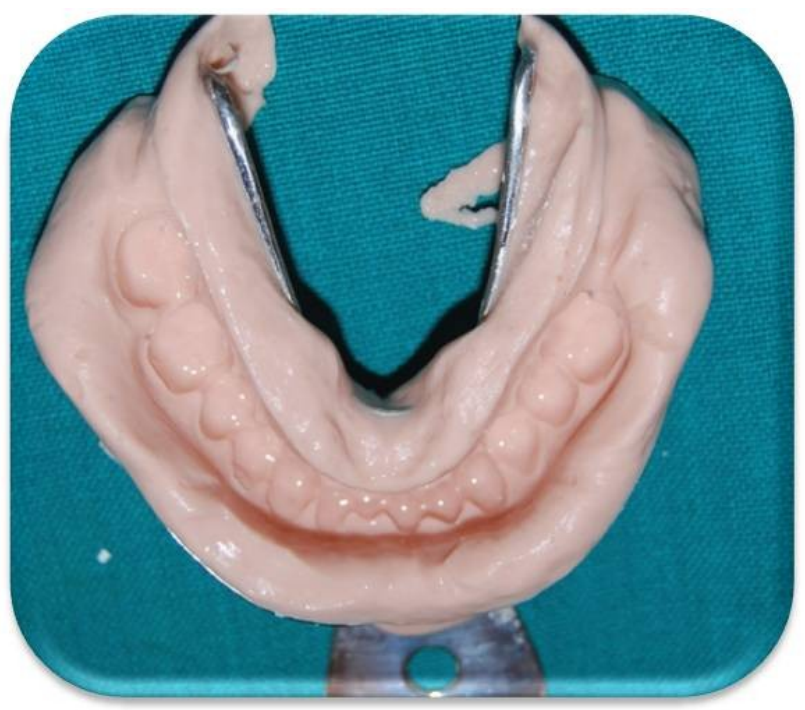

Figure.5(b)MandibularDiagnostic impression

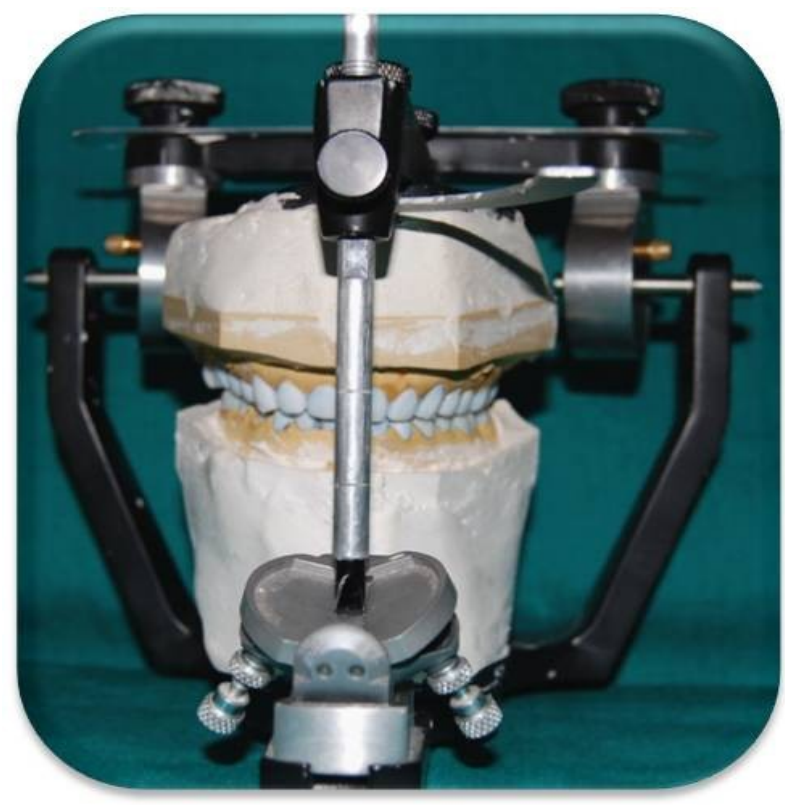

Figure.6 Diagnostic wax-up.

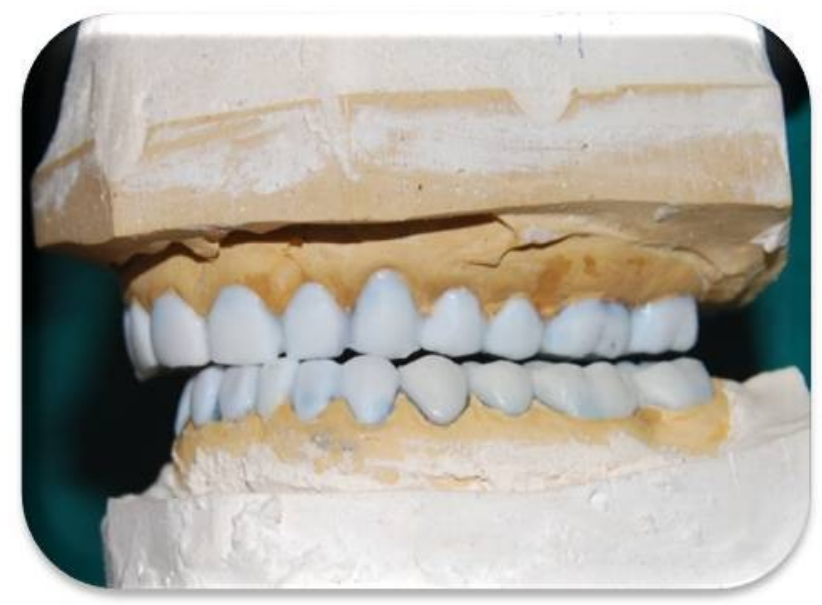

Figure.7Group Function occlusion 


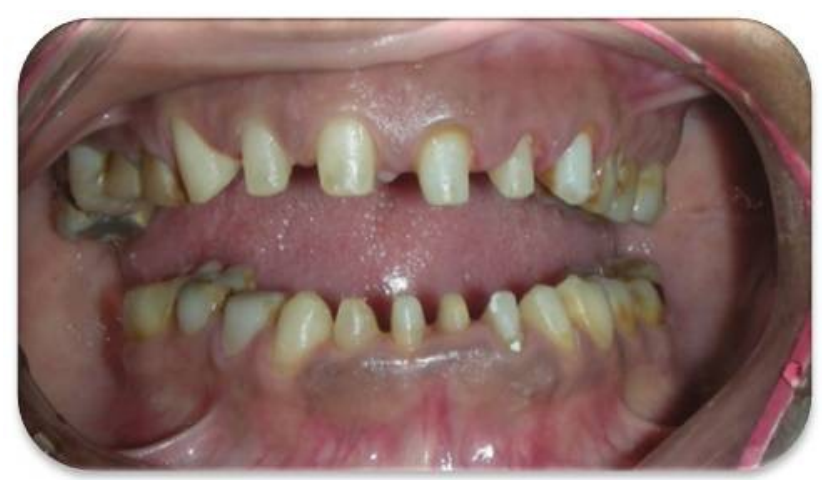

Figure.8 Tooth Preparation

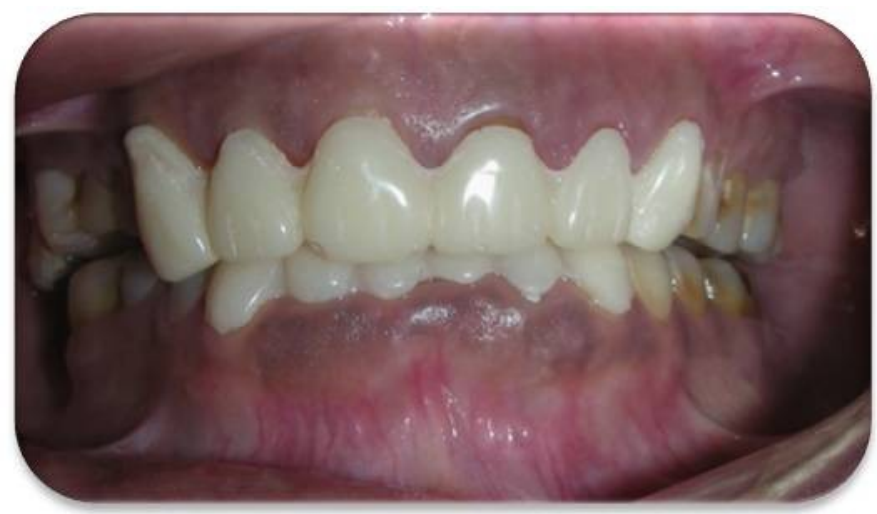

Figure.9Definitive prosthesis 